\section{(2) OPEN ACCESS}

\title{
Whole-body MRI of patients with polymyalgia rheumatica identifies a distinct subset with complete patient-reported response to glucocorticoids
}

\author{
Sarah Louise Mackie, ${ }^{1,2}$ Colin Thomas Pease, ${ }^{3}$ Eiji Fukuba, ${ }^{4}$ Emma Harris, ${ }^{1}$ \\ Paul Emery, ${ }^{1,2}$ Richard Hodgson, 1,2,5 Jane Freeston, 1,2 Dennis McGonagle ${ }^{1,2}$
}

\begin{abstract}
Handling editor Tore K Kvien
- Additional material is published online only. To view please visit the journal online (http://dx.doi.org/10.1136/ annrheumdis-2015-207395).

${ }^{1}$ Leeds Institute for Rheumatic and Musculoskeletal Medicine, Leeds, UK

${ }^{2}$ NIHR-Leeds Musculoskeletal Biomedical Research Unit, Leeds, UK

${ }^{3}$ Leeds Teaching Hospitals NHS

Trust, Leeds, UK

${ }^{4}$ Department of Radiology,

Shimane University, Izumo, Japan

${ }^{5}$ University of Manchester Centre for Imaging Sciences, Manchester, UK
\end{abstract}

\section{Correspondence to}

Dr Sarah Louise Mackie, Leeds Institute for Rheumatic and

Musculoskeletal Medicine, Chapel Allerton Hospital, Leeds LS7 4SA, UK; s.l.mackie@leeds.ac.uk

Received 2 February 2015 Revised 22 June 2015 Accepted 14 July 2015 Published Online First 16 September 2015

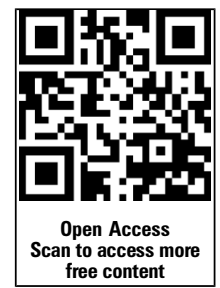

CrossMark

To cite: Mackie $S L$,

Pease $\mathrm{CT}$, Fukuba $\mathrm{E}$, et al.

Ann Rheum Dis

2015;74:2188-2192.

\section{ABSTRACT}

Objectives To determine whether whole-body MRI defines clinically relevant subgroups within polymyalgia rheumatica (PMR) including glucocorticoid responsiveness.

Methods 22 patients with PMR and 16 with rheumatoid arthritis (RA), untreated and diagnosed by consultant rheumatologists, underwent whole-body, multiple-joint MRI, scored by two experts. Patients with PMR reported whether they felt 'back to normal' on glucocorticoid therapy and were followed for a median of 2 years.

Results All patients with PMR were deemed to respond to glucocorticoids clinically. A characteristic pattern of symmetrical, extracapsular inflammation, adjacent to greater trochanter, acetabulum, ischial tuberosity and/or symphysis pubis, was observed in 14/22 of the PMR cases. In PMR, this pattern was associated with complete glucocorticoid response $(p=0.01)$, higher pretreatment $C$ reactive protein (CRP) and serum interleukin- 6 (IL-6), and better post-treatment fatigue and function. Only $1 / 14$ in the extracapsular group could stop glucocorticoids within 1 year, compared with 4/7 of the others. A score derived from the five sites discriminating best between PMR and RA correlated with IL-6 ( $p<0.002)$. IL-6 levels $\geq 16.8 \mathrm{pg} /$ $\mathrm{mL}$ had $86 \%$ sensitivity and $86 \%$ specificity for the extracapsular MRI pattern.

Conclusions A subset of patients with rheumatologistdiagnosed PMR had a characteristic, extracapsular pattern of MRI inflammation, associated with elevated IL-6/CRP and with complete patient-reported glucocorticoid responsiveness.

\section{INTRODUCTION}

Polymyalgia rheumatica (PMR) is a clinically diagnosed cause of glucocorticoid-responsive pain and stiffness at the shoulders and hips, with great variation in the duration of glucocorticoid treatment required. ${ }^{1}$ Previous MRI and 18-fluorodeoxyglucose (FDG)-positron emission tomography (PET) studies have suggested distinct extracapsular ${ }^{3} 4$ or capsularbased $^{5}$ inflammation in PMR. Elevated pretreatment interleukin-6 (IL-6) levels (>10 pg/mL) with good symptomatic response to $20 \mathrm{mg}$ prednisone was associated with requirement for $>1$ year of therapy. ${ }^{6}$ Given the superior resolution of MRI compared with 18-FDG-PET, we sought to determine an anatomical explanation for these findings.
Rheumatologists have traditionally been concerned not to miss rheumatoid arthritis (RA) in patients with PMR, although the anti-citrullinated peptide (anti-CCP) antibody test has made this easier. ${ }^{7} \mathrm{We}$ designed this study to identify patterns of inflammation on whole-body, multiple-joint, 3-Tesla MRI ${ }^{9}$ that distinguished PMR from RA but during follow-up we were struck by the prognostic heterogeneity within the PMR group. Given a known association of ultrasound-defined inflammation with glucocorticoid responsiveness in PMR, ${ }^{10}$ we hypothesised that an extracapsular pattern of inflammation in PMR predicts glucocorticoid response.

\section{METHODS}

Ethical approval was obtained (09/H1307/98, approved by Leeds West Research Ethics Committee 15.1.10; 05/Q1108.28, York Research Ethics Committee). All patients gave written, informed consent.

\section{Cases}

Twenty-two consecutive patients with untreated PMR fulfilling Bird criteria ${ }^{11}$ were identified by two rheumatologists. All had an elevation of at least one acute-phase marker (C-reactive protein (CRP), erythrocyte sedimentation rate (ESR) plasma viscosity (PV)), were negative for rheumatoid factor and anti-CCP antibody and were commenced on prednisolone $15 \mathrm{mg}$ after their MRI scan, increasing to $20 \mathrm{mg}$ at 1-month follow-up if clinically indicated.

Patients recorded pain/stiffness location using mannequins, and graded symptom severity using visual analogue scores (VAS) and Stanford Health Assessment Questionnaire - Disability Index (HAQ-DI). ${ }^{12}$ Patients were asked whether they felt 'back to normal since taking steroids', on a fivepoint Likert scale from 'strongly agree' to 'strongly disagree'. 'Strongly agree' and 'agree' were classified as 'yes', others 'no'. Standardised glucocorticoid taper was adjusted to maintain symptom control until glucocorticoid cessation. ${ }^{2}$ Median follow-up was 2 years.

\section{Imaging controls}

To minimise MRI scorer bias, 16 control MRI scans were chosen from patients with seropositive or seronegative RA. 


\section{MRI}

Whole-body multiple-joint MRI was performed ${ }^{9}$ (see online supplementary methods). Gadolinium was used except where contraindicated. The four non-contrast MRI image files were evaluated (by DM, SLM and EF) to determine presence/ absence of extracapsular PMR pattern as previously described. ${ }^{3}$ All 34 whole-body, multiple-joint, contrast-enhanced MRI image files were anonymised. Axial images were systematically scored in Image in the following order: spine, shoulders, hips, hands, knees, feet. Each defined site was semiquantitatively consensus scored by the two experts (DM and EF), scoring 0 , 1 , 2 or 3 , for no inflammation, mild, moderate or severe inflammation respectively (figure 1). Each MRI was also classified as 'extracapsular pattern' or 'non-extracapsular pattern'. The anonymisation code was not broken until the MRI scoring datasheet (including overall classification) had been locked down.

\section{IL-6 measurement}

IL-6 was measured by ELISA (IL-6 Quantikine, R+D Systems) using serum taken from consenting patients before MRI.

\section{Analysis}

We tested the hypothesis that an extracapsular pattern of disease was associated with glucocorticoid responsiveness. Statistical analyses were performed in SPSS V.21 (IBM).

\section{RESULTS}

\section{Demographics and disease characteristics}

At screening, all 22 patients with PMR fulfilled Bird criteria ${ }^{11}$ and (retrospectively) the provisional ACR/EULAR classification criteria, ${ }^{8}$ including elevation of at least one acute-phase marker; in two PMR cases, however, acute-phase markers normalised by the time the MRI scan was done. At follow-up, PMR was confirmed as the most likely diagnosis. All 22 patients were recorded by the treating rheumatologist as responding to prednisolone; in three cases an increase in dose was required for complete response. No alternative explanation for patients' musculoskeletal symptoms was found.

\section{Training set (non-contrast) MRIs in PMR}

Extracapsular inflammation ${ }^{3}$ was seen in 2/4 non-contrast MRI scans of patients with PMR. Oedema was seen around the
Grade 0

Peri-
acetabular

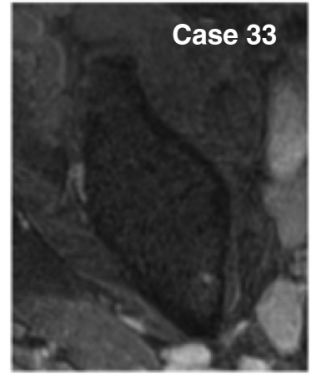

Hip

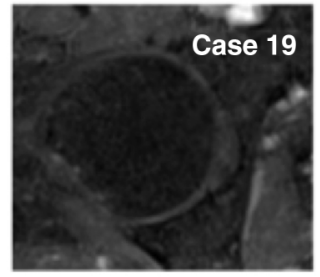

Greater trochanter
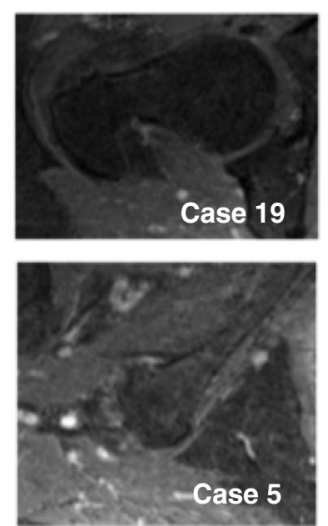

Ischial tuberosity

\section{Inferiou to symphysis pubis}

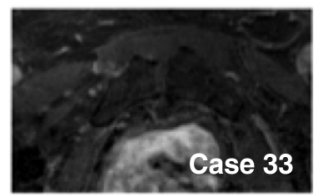

Grade 1
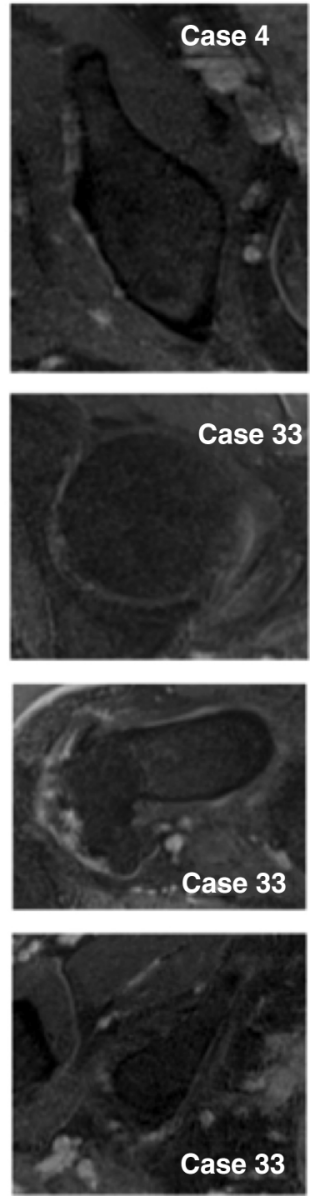

Grade 2
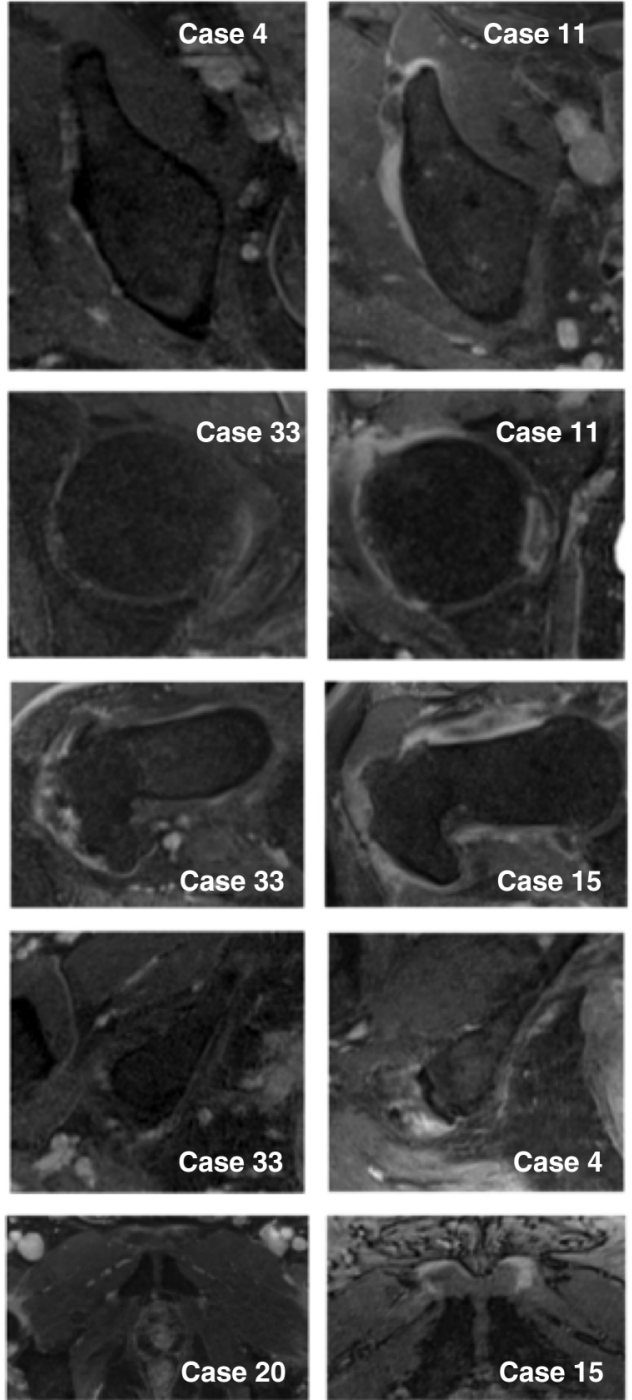

Grade 3
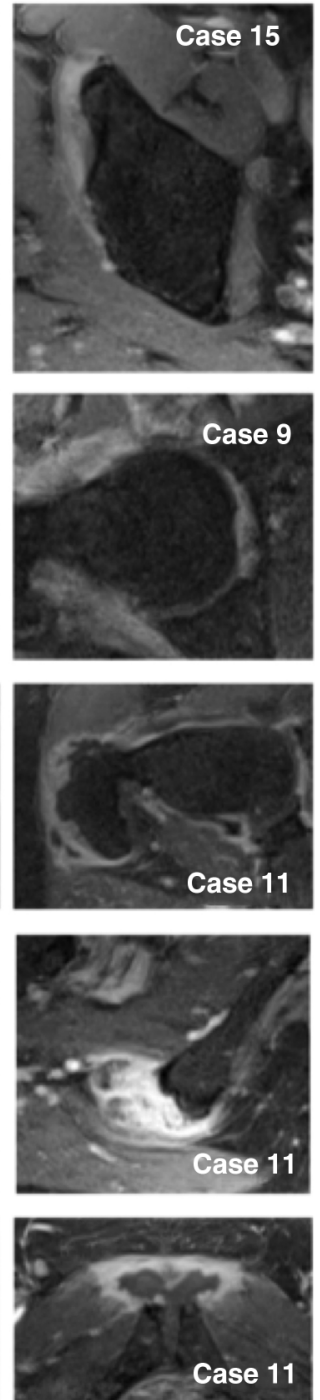

Figure 1 Exemplar images of semiquantitative scoring system. 
greater trochanter in both, and of subdeltoid bursa, glenohumeral joint and below the symphysis pubis in one. There was no difference between the patients with PMR who did and did not receive contrast (see online supplementary table S2).

\section{Gadolinium-enhanced whole-body multiple-joint MRI}

The same extracapsular pattern was seen on contrast MRI as with non-contrast MRI. Inflammation around the shoulders was seen in both PMR and RA; PMR additionally featured pelvic inflammation especially adjacent to greater trochanter and ischial tuberosity (see online supplementary figure S1) and periacetabular anterolateral to the rim of the acetabulum, without involving the synovial hip joint and extending superiorly from the anterior hip capsule, medial to gluteus minimus and lateral to the iliac bone, not typical for iliopectinal (iliopsoas) bursitis (see online supplementary figure S1). We additionally observed inflammation inferior to the symphysis pubis (see online supplementary figure S1). Bone oedema was absent. 14/22 of the patients with PMR and $1 / 16$ of the patients with RA were classified as 'extracapsular pattern' $(p<0.001)$. Results of semiquantitative scoring (figure 1 ) are shown in figure 2.

\section{Clinical associations of extracapsular pattern}

Prior to glucocorticoid therapy, according to the rheumatologists' assessments, the extracapsular group and the non-extracapsular group appeared to have the same clinical syndrome of PMR with no difference in the PMR-AS activity score. ${ }^{13}$ The nonextracapsular group was all women, and had a lower CRP and IL-6 (table 1). Males had a higher MRI inflammation score $(p=0.046)$ and a lower stiffness VAS $(p=0.037)$, non-significant after Bonferroni correction (data not shown). 9/13 of those scoring 2 or 3 at ischial tuberosity indicated buttock pain on pain/stiffness mannequins, compared with $0 / 5$ of the remainder (sensitivity 69\%, specificity 100\%) (see online supplementary figure S3).

Where questionnaire data were available from first follow-up (not done in three because of time constraints), 11/13 of those with characteristic 'extracapsular pattern' of MRI inflammation, and $1 / 6$ of the remainder, stated that they felt 'back to normal' since taking steroids $(p=0.01)$. This was reflected in better patient-reported function (HAQ-DI) and fatigue VAS (table 1) but no difference in post-treatment pain or stiffness VAS between patients with and without the extracapsular pattern (data not shown). The remaining three patients were later asked whether they had felt 'back to normal' in the first month after taking steroids; therefore, $12 / 14$ of all the patients with PMR an extracapsular pattern felt 'back to normal', compared with $1 / 8$ of those without an extracapsular pattern $(p=0.001)$. Those with an extracapsular pattern were less likely to be able to stop glucocorticoid therapy within the first year.

Figure 2 Results of blinded scoring of gadolinium-enhanced MRI scans. Prosthetic joints are treated as missing data (cells left blank). GC, glucocorticoid. 
Table 1 Description of features of patients with polymyalgia rheumatica (PMR) with and without characteristic extracapsular pattern of inflammation

\begin{tabular}{|c|c|c|c|}
\hline & Extracapsular pattern $(n=14)$ & Non-extracapsular pattern $(n=8)$ & $\mathrm{p}$ Value \\
\hline \multicolumn{4}{|l|}{ Demographics } \\
\hline Age, median (range) & $75(55,85)$ & $78(70,84)$ & 0.22 \\
\hline Male, $n(\%)$ & $8(57 \%)$ & $0(0 \%)$ & 0.02 \\
\hline \multicolumn{4}{|l|}{ Acute-phase markers } \\
\hline ESR, median (range), $\mathrm{mm} / \mathrm{h}$ & $46(9,119)$ & $38(4,81)$ & 0.63 \\
\hline CRP, median (range), mg/L & $36(5,118)$ & $5.25(5,76)$ & 0.03 \\
\hline PV, median (range), $\mathrm{mPa} \mathrm{s}$ & $1.93(1.78,2.12)$ & $1.81(1.57,2.04)$ & 0.36 \\
\hline IL-6, median (range), pg/mL & $25.8(0.3,87.6)$ & $6.0(0.2,131.5)^{*}$ & 0.04 \\
\hline \multicolumn{4}{|l|}{ Composite disease activity scores } \\
\hline PMR-AS (median, IQR) & $78.6(53.8,103.0)$ & $70.2(34.4,106.1)$ & 0.73 \\
\hline \multicolumn{4}{|l|}{ Pretreatment patient-reported outcomes } \\
\hline Pain VAS (median, IQR) & $7.7(5.0,8.1)$ & $7.9(4.3,8.7)$ & 0.63 \\
\hline Stiffness VAS (median, IQR) & $6.3(4.5,8.0)$ & $8.2(5.2,8.9)$ & 0.29 \\
\hline Fatigue VAS (median, IQR) & $7.1(5.2,7.6)$ & $8.4(7.0,9.7)$ & 0.03 \\
\hline HAQ-DI (median, IQR) & $1.25(1.09,1.50)$ & $1.56(1.28,2.09)$ & 0.07 \\
\hline \multicolumn{4}{|l|}{ Assessment of glucocorticoid responsiveness at first follow-up } \\
\hline 'I feel back to normal since taking steroids'. $\mathrm{n}(\%)$ & $11 / 13(85 \%)$ & $1 / 6(17 \%)$ & 0.01 \\
\hline 'I feel [or felt] back to normal since taking steroids'. n (\%) & $12 / 14(86 \%)$ & $1 / 8(13 \%)$ & 0.001 \\
\hline Fatigue VAS at follow-up (median, IQR) & $1.3(0.2,3.8)$ & $7.1(3.6,9.8)$ & 0.02 \\
\hline HAQ-DI at follow-up (median, IQR) & $0(0,0.625)$ & $1.0(0.76,2.07)$ & 0.003 \\
\hline \multicolumn{4}{|l|}{ Prognosis } \\
\hline Stopped glucocorticoids permanently after $<1$ yeart & $1 / 14$ & $4 / 7$ & 0.03 \\
\hline Relapse-freet & $7 / 14$ & $2 / 8$ & 1.00 \\
\hline Relapsed when on $5 \mathrm{mg}$ or moret & $3 / 14$ & $3 / 7$ & 0.35 \\
\hline Required initial dose increase $>15 \mathrm{mg}$ & $2 / 14$ & $1 / 8$ & 1.00 \\
\hline \multicolumn{4}{|c|}{$\begin{array}{l}\text { The PMR-AS is the PMR Activity Score as described by Bird and Leeb. Either Mann-Whitney U test or Fisher's exact test was used for non-normally distributed values; unpaired t test for } \\
\text { normally distributed variables. All tests were two-tailed. Apart from glucocorticoid responsiveness (the a priori hypothesis), p values should be interpreted in the light of multiple testing. } \\
\text { Bonferroni correction for all the variables reported here (likely over-stringent because of strong correlation between ESR/CRP/PV/IL-6 and between patient-reported VAS scores) would } \\
\text { require a threshold of } 0.05 / 19 \text {, or } p<0.0026 \text {. } \\
\text { *Excludes one patient who did not have IL-6 measured. } \\
\text { tExcludes one patient who was lost to follow-up at } 4 \text { months. } \\
\text { CRP, C-reactive protein; IL-6, interleukin-6; PV, plasma viscosity; VAS, visual analogue score. }\end{array}$} \\
\hline
\end{tabular}

The two patients with PMR extracapsular pattern who were not complete glucocorticoid responders by self-report (figure 2) had the highest IL- 6 and CRP, and both required escalation of prednisolone dose for full response. Another patient with extracapsular pattern later developed biopsy-proven giant cell arteritis.

\section{Association of IL- 6 with MRI inflammation}

The top five MRI features (mean of left and right) were summed to provide a composite score. This was significantly associated with IL-6 $(p<0.001)$ (see online supplementary figure S2) but not with CRP $(p=0.055)$. The most discriminatory IL-6 cut-off for the extracapsular pattern was $\geq 16.8 \mathrm{pg} / \mathrm{mL}$ (sensitivity $86 \%$, specificity $86 \%)$.

\section{DISCUSSION}

All our patients were diagnosed with PMR by rheumatologists; we sought to determine whether this could be further stratified based on the pattern and extent of inflammation on whole-body MRI. We identified a subset, with characteristic, extracapsular pattern of inflammation on MRI that was more likely to feel 'back to normal' after glucocorticoids. MRI allowed good resolution of pelvic inflammation. In addition, despite having more males (male gender in PMR generally predicts shorter glucocorticoid duration ${ }^{14}$ ), our 'extracapsular' group was also more likely to require glucocorticoid treatment for $>1$ year. IL- 6 correlated with pelvic MRI inflammation; a cut-off of $\geq 16.8 \mathrm{pg} / \mathrm{mL}$ IL-6 had $86 \%$ sensitivity and $86 \%$ specificity for the extracapsular pattern. Our data support an extra-articular model of the primary inflammatory change in $\mathrm{PMR}^{3}$ A recent report describes focal 18-FDG-PET uptake anterior to the hip joint in $\mathrm{PMR}^{15}$ similar to our 'periacetabular' pattern.

Strengths of this study include the standardised assessments and the blinded MRI scoring. PMR diagnoses were all made by a consultant rheumatologist, and all patients were treated as PMR without any alternative diagnosis supervening.

The limitations of this study were its descriptive and exploratory nature, small numbers, slightly younger age of the RA group, and the subjectivity inherent in clinical diagnosis of PMR even following diagnostic guidelines. ${ }^{8} 16$ We hypothesise the 'non-extracapsular' patients with PMR may be a pathogenetically heterogeneous group, analogous to 'autoantibody-negative RA'. Whether they ought to be labelled PMR is a philosophical question beyond the scope of this investigation.

This novel, pathoanatomical description of the clinical spectrum of PMR adds weight to the idea of PMR as a clinically heterogeneous disorder. ${ }^{1}$ MRI may help to identify a more homogeneous subset, with potential value for defining eligibility for early clinical trials of targeted therapies. Our data suggest that in the specialist setting CRP and IL-6 may be more 
prognostically useful tests than ESR or PV. An MRI might be useful in cases of diagnostic doubt. The diagnostic importance of glucocorticoid responsiveness is still debated in $\mathrm{PMR}^{8} 17$ especially since the best way to measure response remains unclear: the limitations of previously proposed disease activity scores, including the physician global assessment and the PMR-AS, have been well-discussed elsewhere. ${ }^{10}$ Although most disease activity scores focus on pain and stiffness, we identified residual fatigue and functional impairment after glucocorticoid treatment in our non-extracapsular group; this is of interest since fatigue ${ }^{18}$ and disability ${ }^{19}$ have been identified from recent qualitative studies as being important to patients. We found that MRI yielded additional valuable information to the clinical assessment, particularly in the pelvic region where an extracapsular pattern was clearly seen in a distinct subset of patients. Further research is required to determine the clinical utility of MRI, symptom location (eg, buttock pain) or CRP/IL-6 as diagnostic, prognostic or treatment stratification features in PMR.

Twitter Follow Sarah Mackie at @Sarah_L_Mackie

Acknowledgements Thanks to Ged Connolly-Thompson for anonymising the case-control MRIs, allocating random anonymisation codes and keeping the decode sheet until the scoring sheet had been locked down. Rob Evans and Carole Burnett performed the MRI scans, and Tracey Hulland provided administrative support and welcomed the patients. Thanks to Farah Mohamad Idris for carefully going through the pain and stiffness mannequins, digitally superimposing the images to give combined pain/stiffness images, and comparing with the MRI scoring data. Thanks to Agata Burska for assistance with ELISAs and Professor Ann Morgan for advice on earlier versions of the manuscript. Permission has been obtained from all persons named in the acknowledgement. SLM had full access to all the data in the study and takes responsibility for the integrity of the data and the accuracy of the data analysis.

Contributors SLM helped design the study, conducted research visits, organised MRI scoring, analysed data, and drafted the paper; CTP recruited patients, collected clinical data; EF helped devise the scoring system and scored MRIs; EH carried out the IL-6 ELISAs; PE and JF led the Disease Continuum study, and provided clinical data on the patients with RA; RH developed the MRI protocol; DM helped design the study, recruited patients and scored the MRIs. All authors contributed to design and/or data interpretation, revised the manuscript for important intellectual content, and approved the final version.

Funding SLM was funded by a National Institute for Health Research Academic Clinical Lecturer Award during the data collection and is currently funded by a National Institute for Health Research Clinician Scientist Award. MRI scans were funded by the NIHR Leeds Musculoskeletal Biomedical Research Unit. Biomarker analyses were funded by a grant to SLM from the Leeds Teaching Hospitals Charitable Trustees. This article presents independent research supported by the National Institute for Health Research (NIHR). The views expressed are those of the authors and not necessarily those of the NHS, the NIHR or the Department of Health. An earlier version of this work was presented in poster form at the American College of Rheumatology Annual Meeting in November 2014.

Competing interests None declared.

Ethics approval Leeds West Research Ethics Committee and York Research Ethics Committee.

Provenance and peer review Not commissioned; externally peer reviewed.

Data sharing statement Further data including anonymised MRI images available to bona fide researchers by request to the corresponding author. Any data that could identify individual patients will not be available, for ethical reasons.
Open Access This is an Open Access article distributed in accordance with the terms of the Creative Commons Attribution (CC BY 4.0) license, which permits others to distribute, remix, adapt and build upon this work, for commercial use, provided the original work is properly cited. See: http://creativecommons.org/ licenses/by/4.0/

\section{REFERENCES}

1 Hutchings A, Hollywood J, Lamping DL, et al. Clinical outcomes, quality of life, and diagnostic uncertainty in the first year of polymyalgia rheumatica. Arthritis Rheum 2007;57:803-9.

2 Mackie SL, Hensor EM, Haugeberg G, et al. Can the prognosis of polymyalgia rheumatica be predicted at disease onset? Results from a 5-year prospective study. Rheumatology (Oxford) 2010;49:716-22

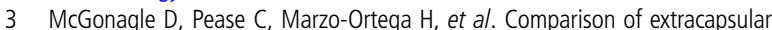
changes by magnetic resonance imaging in patients with rheumatoid arthritis and polymyalgia rheumatica. J Rheumatol 2001;28:1837-41.

4 Mori S, Koga Y, Ito K. Clinical characteristics of polymyalgia rheumatica in Japanese patients: evidence of synovitis and extracapsular inflammatory changes by fat suppression magnetic resonance imaging. Mod Rheumatol 2007;17:369-75.

5 Cimmino MA, Camellino D, Paparo F, et al. High frequency of capsular knee involvement in polymyalgia rheumatica/giant cell arteritis patients studied by positron emission tomography. Rheumatology (Oxford) 2013;52:1865-72.

6 Weyand CM, Fulbright JW, Evans JM, et al. Corticosteroid requirements in polymyalgia rheumatica. Arch Intern Med 1999;159:577-84.

7 Pease CT, Haugeberg G, Morgan AW, et al. Diagnosing late onset rheumatoid arthritis, polymyalgia rheumatica, and temporal arteritis in patients presenting with polymyalgic symptoms. A prospective longterm evaluation. J Rheumatol 2005;32:1043-6.

8 Dasgupta B, Cimmino MA, Maradit-Kremers H, et al. 2012 provisional classification criteria for polymyalgia rheumatica: a European League Against Rheumatism/ American College of Rheumatology collaborative initiative. Ann Rheum Dis 2012;71:484-92.

9 Freeston J, Conaghan P, Grainger A, et al. Usefulness of novel whole body multiple joint MRI imaging in establishing accurate and timely diagnoses in patients presenting with inflammatory arthritis. Rheumatology (Oxford) 2012;51(Suppl 3): iii68.

10 Matteson EL, Maradit-Kremers H, Cimmino MA, et al. Patient-reported outcomes in polymyalgia rheumatica. J Rheumatol 2012;39:795-803.

11 Bird HA, Esselinckx W, Dixon AS, et al. An evaluation of criteria for polymyalgia rheumatica. Ann Rheum Dis 1979:38:434-9.

12 Kalke S, Mukerjee D, Dasgupta B. A study of the health assessment questionnaire to evaluate functional status in polymyalgia rheumatica. Rheumatology (Oxford) 2000:39:883-5.

13 Leeb BF, Bird HA. A disease activity score for polymyalgia rheumatica. Ann Rheum Dis 2004;63:1279-83.

14 Dejaco $C$, Singh $Y$, Perel $P$, et al. Current evidence on prognostic factors in patients with polymyalgia rheumatica (PMR): a systematic literature review informing the ACR/EULAR recommendations for the management of PMR. Ann Rheum Dis 2014;73(Suppl 2):553.

15 Takahashi $\mathrm{H}$, Yamashita $\mathrm{H}$, Kubota $\mathrm{K}$, et al. Differences in FDG PET/CT between EORA and PMR. Mod Rheumatol 2015:25:546-51.

16 Dasgupta B, Borg FA, Hassan N, et al. BSR and BHPR guidelines for the management of polymyalgia rheumatica. Rheumatology (Oxford) 2010;49:186-90.

17 Spiera R, Westhovens R. Provisional classification [corrected] criteria for polymyalgia rheumatica: moving beyond clinical intuition? Arthritis Rheum 2012;64:955-7.

18 Mackie SL, Hughes R, Walsh M, et al. "An impediment to living life": why and how should we measure stiffness in polymyalgia rheumatica? PLOS ONE 2015;10: e0126758.

19 Twohig H, Mitchell C, Mallen C, et al. "I suddenly felt I'd aged": a qualitative study of patient experiences of polymyalgia rheumatica (PMR). Patient Educ Couns 2015;98:645-50. 